
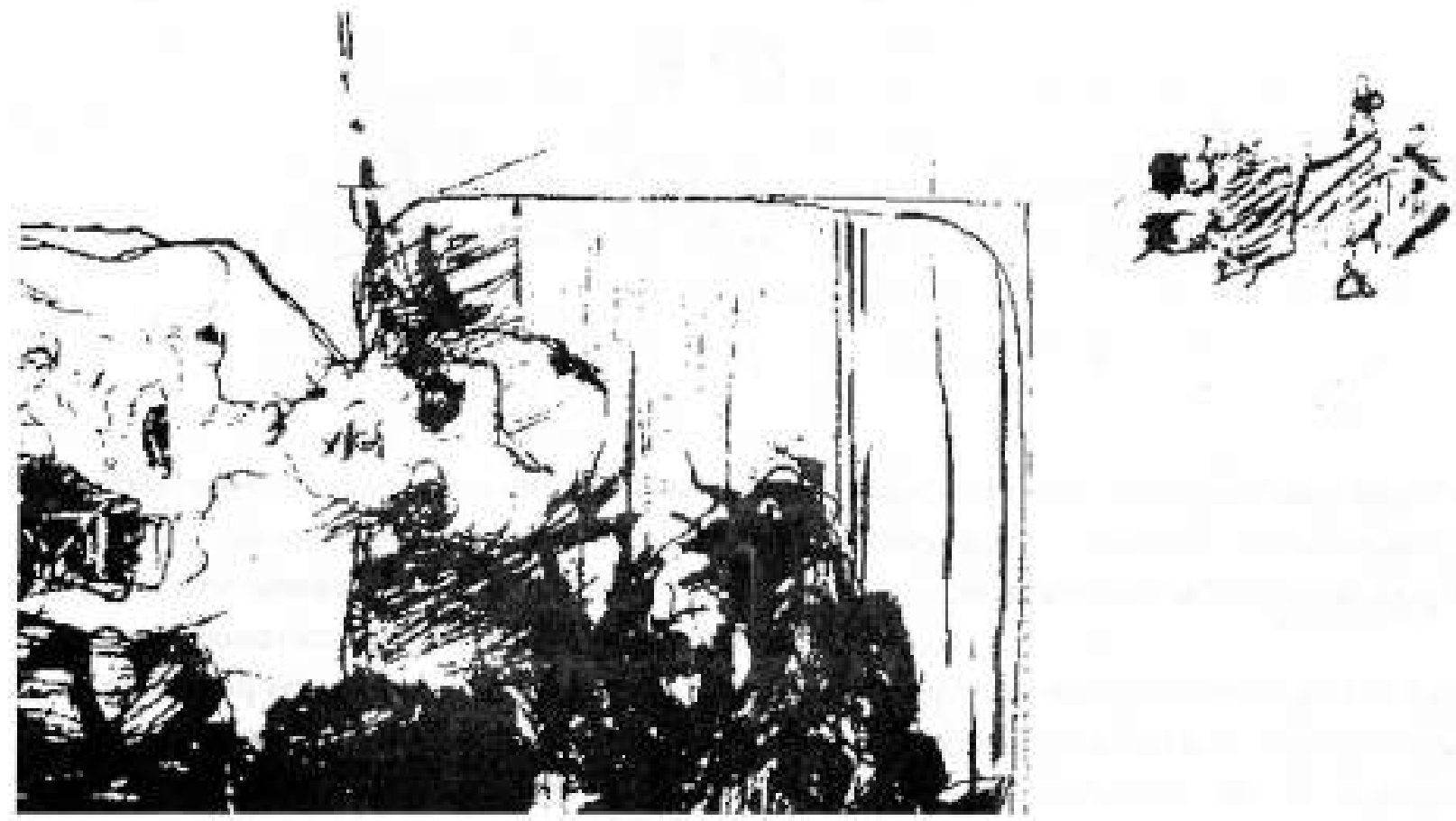

\title{
ELEMENTOS PARA COMPRENDER EL SENTIDO DE LA PROYECCIÓN UNIVERSITARIA EN LA UNIVERSIDAD AUTÓNOMA DE MANIZALES
}

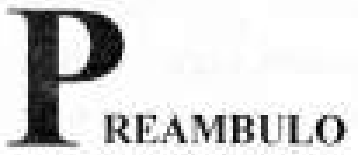

IOSÊ. RUBEN CASTHLLOGARCIA

Trabajador Social Magister en Desarrollo Educativo y Social

Profesor Titular Facultad de Fisisterapia Universidad Autónoma de Manizales
REAMBULO

El presente documento expresa el punto de vista de la Vicerrectoria de Investigaciones y de Proyección Universitaria de la Universidad Autónoma de Manizales, (U.A.M)', es el resultado de sistematizar algunos puntos de vista de diferentes autores y de las experiencias que hemos tenido al respecto. Se asume como un texto de trabajo. susceptible de ser 


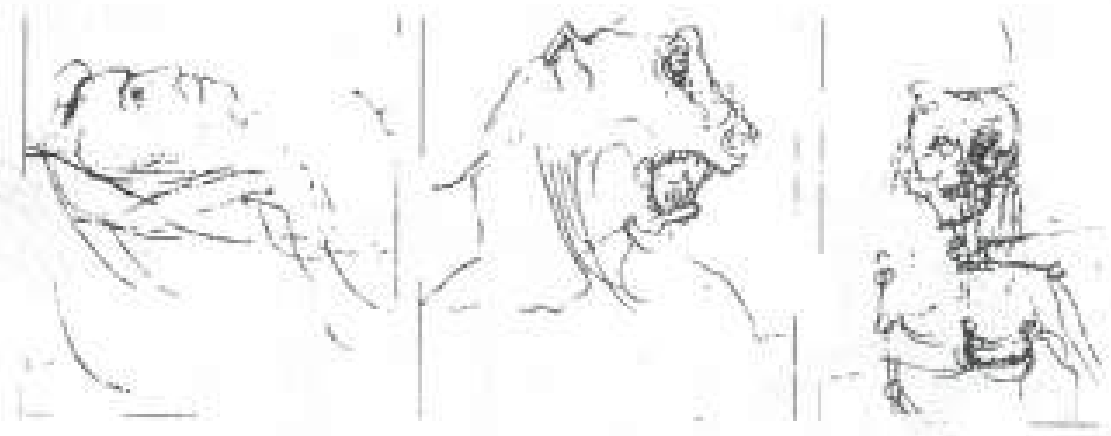

modificado con base en las opiniones de quienes estén interesados en la temática y nos sentiremos muy gustosos de recibir sus comentarios.

A manera de comienzo. se puede decir que cuando se hatce referencia a las funciones sustantivas de la universidad, es usual que en el ămbito académico se indique que éstas son la docencia, la investigación y la extensión. Sin embargo, al definirlas se percibe la existencia de diversos puntos de vista acerca de los alcances y sentidos que se le dan a estos términos. En el presente documento, se plantean algunos criterios que buscan contribuir a pensar los sentidos que se le dan a uno de esos conceptos, el de extensión. con la intención de que esta Vicerrectoria asuma posición al respecto. en la perspectiva de orientar las acciones relacionadas con la Proyección Universitaria.

Cuando se habla de extensión se perciben tres conceptos: El propio de extensión. la proyección social y la proyección universitaria. En razón de ello. hasurgido la necesidad de lograt claridad en relación con el sentido que se le da a cada uno de ellos, veamos:

Antes de avanzar se hace importante anotar que en principio esto podria aparecer como un asunto del uso de términos. sin embargo. es de tener en cuenta que las diversas concepciones que se tienen al respecto. condicionan las posiciones que se adopten a nivel institucional, dado que de ello se desprenden orientaciones, opciones. puntos de vista y procedimientos en los que se apoya suacción y por ende las repercusiones que esto pueda tener en lo que se realice. Algunos de los puntos de vista implicados derivan de los conceptos de educación. sociedad, persona, cultura, el papel de la universidad, las relaciones sociedad - universidad y pedagogia. Además, como se hace obvio, de aquello que se plantee derivarán las situaciones y problemas que se deben atender. las formas metodológicas a utilizar y las prácticas a desarrollar. Es de aclarar que la pretensión de este texto llega basta proponer algunos puntos de vista que puedan ayudar a comprender el sentido de la Proyección Universitaria en la Universidad Autónoma de Manizales y los alcances que eilo tiene en la vida de la comunidad, de la cual somos parte.

La razón de ser de la temática tiene que ver con el desarrollo del sistema educativo en general : especificamente de la universidad como institución. teniendo como base las funciones que históricannente les han sido asignadas dentro de su competencia institucional. por parte de la sociedad en que se origina y del Estado que legisla sa campo de acción. Un aspecto que se hace común al tratar el tema se refiere a la relación que establecen las institucionss academicas y el conocimiento que manejan con respecto de la sociedad en la cual realizan sus funciones y de las repercusiones que tienen estos 
saberes en la vida de las poblaciones con las cuales se relacionan. Veamos algunas ideas al respecto:

\section{ANTECEDENTES GENERALES}

A manera de resumen. se puede decir que en las sociedades contemporáneas, relacionadas con el modo de producción capitalista se ha presentado una acentuada especialización en el desempeño de las funciones, tanto de las instituciones como de las personas, esto como consecuencia de la-creciente divisiòn social del trabajo. La eficacia. la efectividad y laeficiencia en la producción. privilegia el interés por obtener mayor cantidad de productos a menor costo para efectos de lograr mejores rendimientos con las inversiones que se hagan. En términos de los fundamentos de este sistema de vida, se trata de asegurar la ganancia suficiente que garantice el incremento de los capitales invertidos. Para ello, el funcionamiento social debe estimular el desarrollo de las habilidades y las destrezas particulares que se hacen necesarias para satisfacer las demandas del mercado,

Como consecuencia de lo anterior, la sociedad actual se ha fragmentado en diversidad de protesiones, ocupaciones y oficios, destinados a satisfacer las múltiples necesidades de la población. Uno de estos aspectos es la educacion. otros la salud. el empleo. la vivienda, etc.
Veamos lo que ba venido sucediendo con la especialización en relación con la educación. vista desde lo que se ha denominado como la educación tradicional. que se ha llevado a cabo en las instituciones destinadas para ello:

-La educación, antes responsabilidad de la comunidad, es trasladada a algunas instifuciones especificas a las cuales se les asigna la funcion de capacitav, instruir $y$ de formar a los individuos, para que ingresen paulatinamente a la vida social.

En estas condiciones, a fa escuela se le ha encargado la labor de la preparación de las nuevas generaciones: monopolizando lo que tiene que ver con la enseñanza y con el aprendizaje $\mathrm{c}$ intentando reemplazar a la sociedad en lo concernienste con su mision reproductiva. Ejemplo de esto es la familiat. la cual cuda vez es menos eficax en el desarrollo de dicha labor, puesto gue la custodia de los nifos, la construcción del pensamiento, normas, valores y el desarrollo de conocimientos, habilidades y destrezas, han sido asumidas paulatinamente por la escuela. En otros términos, la teducacion se ha venido reduciende como proceso de socializacion y lo ha verido dando paso a la escolarizacion.

La escolaridad ha distanciado la laber que cumple la escuela y los procesos edicativas que ocurren en el acontecer cotidiano de las colectividades lumaras. La escolaridad ha convertido la educacion en instraceión y transmisión de informacion, lahor que en muchos casos se hace en forma esquematica y rutinaria. Desconoce que los oducandos son seres individuales e historicos, se les despersmifica. los trata como universales. homogéneos y susceptibles de desarrollar mediantis acciones programadas quic se desartollan en lugares en donde se acrua al maryen de la realidad.

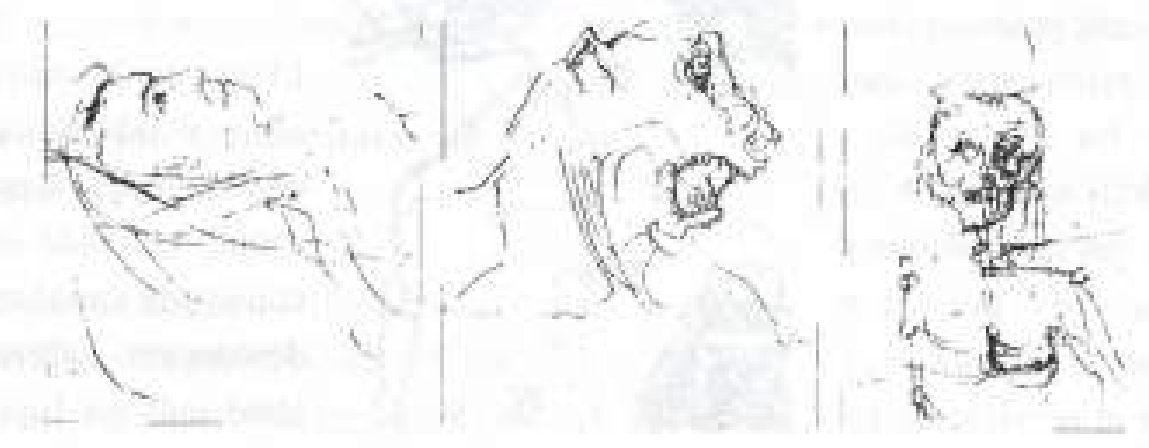


De esta manera, la labor de formación y de construcción del hombre, propia de la educación ha sido reemplazada por la instrucción. Esta situación ha llevado a entender la necesidad de transformar la manera de orientar la educacion":-
Si se percibe la necesidad de pensar otras formas de orientar la educación. ello trae consigo criterios y prácticas diferentes de asumir las relaciones entre las instituciones académicas con respecto de la sociedad en la cual se desempeña. En principio, se requiere expresar los conceptos de educación en que se apoya tanto el sistema educativo ancestral como las nuevas perspectivas.

\section{PERSPECTIVASEDUCATIVAS}

En razón de lo planteado se puede evidenciar que las concepciones que orientan la labor educativa. condicionan el sentido que se le plantea a las relaciones entre las instituciones academicas y la sociedad.

Si se apoya en la concepción en la cual se fragmenta lo social. cada institución desamolla sus funciones independientemente, tratando de lograr determinadas competencias entre los beneficiarios del servicio, teniendo como base sus propias funciones y en el mejor de los casos buscando acercamientos con otras entidades con la intención de complementar su labor. Desde este punto de vista. la intención bảsica de la labor educativa se ha orientado a capacitar. es decir a instruir a los estudiantes en las habilidades y destrezas que les permitan desempenarse eficaz $y$ eficientemente al servicio de las demandas de la sociedad. Esta manera de ver las cosas, privilegia la instrucción de las personas en los saberes requeridos por las profesiones y oficios en los cuales se da la educación.

Otra forma de ver el fenómeno educativo considera que el desarrollo de las sociedades y de los individuos es integral, y por lotanto. las instituciones hacen parte de ella, y en esa calidad. la labor que realiza con los estudiantes debe responder a las necesidades de la sociedad y contribuir a las soluciones que ella demanda. de ahi que no se trata de acercamientos sino de compromisos y de responsabilidades con el colectivo.

En el primer punto de vista (fragmentación social). se pueden diferenciar dos matices: De un lado aquel que segrega tajantemente las instituciones educativas del funcionamiento de la sociedad, la plantean como el centro del saber y modernamente proponen la utilidad de éste en razón de suaplicabílidad, es decir. la institución tiene sentido en la

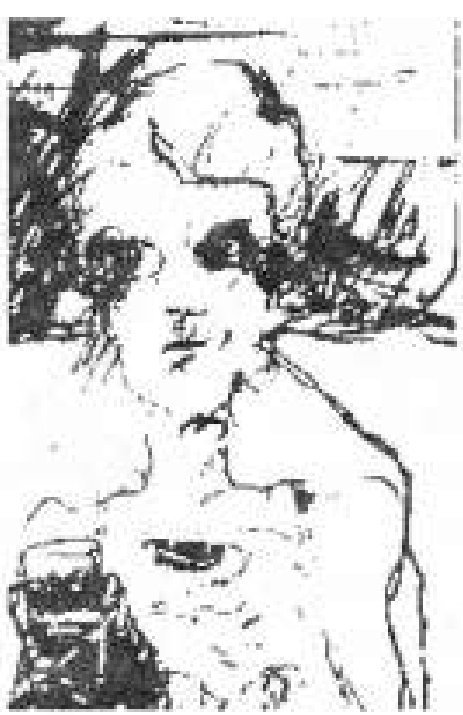
medida en que presta o vende el servicio de lo educativo, es por ello que su labor se orienta exclusivamente a ello. Pedagogicamente se centran en la profesionalizacion.

El otro mattz. más contemporáneo. asume esta labor aún como un centro educativo que tiene que ver con lo social, entiende este componente como una variable que no se debe desconocer. Este reconocimiento se hace más en funciōn de aceptar 
conceptualmente que la institución está inmersa y ejerce en un medio que le contextúa y que le toca su acción.

Ambas posturas no logran asumir lo sacial como parte de un compromiso que

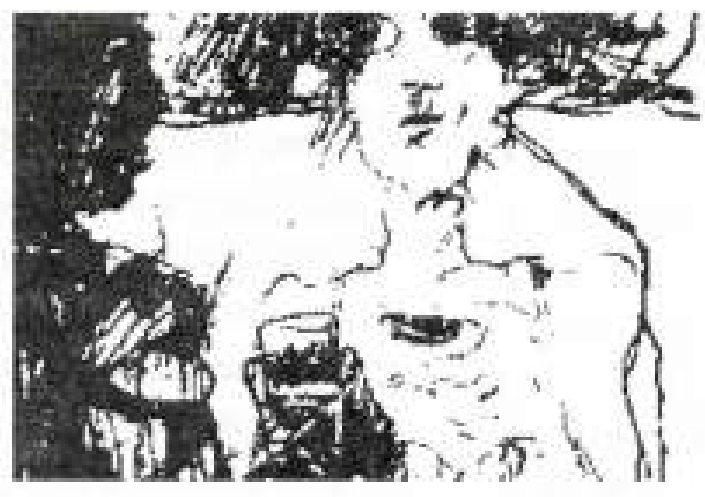
deriva de la pertenencia a la sociedad, dado que su pretensión se apoya en reconocer que se trata de ampliar la visión educativa. de posibilitar otros puntos de referencia para complementar la formación académica. aqui lo social se entiende como un ayregado para la educación y por ende, las acciones que se originan en las instituciones académicas se ven como resultado de una visión filantrópica. Esta forma de mirar lo social y lo educativo, dio origen a las actuales propuestas de "Extensión Universitaria". pensadas como una forma de construir vinculos con la realidad social y de dar mayor alcance a su labor.

Con respecto de la visión que percibe la realidad como algo integral, se puede destacar lo planteado en la Facultad de Fisioterapia. en un documento en el cual se sustenta conceptualmente cl área de Proyección Comunitaria. en momentos en que aún se requeria de mostrar las razones de orientar procesos educativos que trascendieran la función universitaria de "Extensión" y que se pensara en términos de "Proyección", a la luz de las condiciones historicas presentes en ese momento en nuestro pais y que aún pueden tener vigencia, veamos:

\section{-Por qué la Proyección Comunitaria en la}

\section{Educación actual?}

Los procesos de Descentralizacicin Administrativa. Politica y Fiscal. en los cuales se ha venido involucrando la sociedad colombiana desde comienzos de la decada de los âtos $80^{\prime}$, nos invitan constantemente a promover la construcción de una sociedad en la cual se fomente el pluralismo, la convivencia ciudadana. la participación y la búsqueda de soluciones colectivas a las necesidades de la población.

Las normas (leyes. decretos, etc.) que se han elaborado. especialmente desde 1986, han contribuido a generar un marco de referencia importante para orientar el desarrollo social del pais. Se pone al orden del dia la necesidad de generar procesos de acción social basados en la autonomia local, en la búsqueda del mejoramiento efectivo de la calidad de vida de la población y de la construcción de espacios que favorezan el desarrollo del hombre como tal. La prestación de los servicios sociales por parte del Estado hacia la población ha sido reglamentado en el mismo sentido, tal es el caso de sectores como la educación y la salud.

Por lo anterior. hoy en dia se hace imponante tener en cuenta que los aspectos relacionados con la práctica educativa deben tener relación directa con la vida cotidiana de los participantes en ellos. Por lo tanto. las personas que realicen actividades de formación académica deben orientar los contenidos que les sirven de base al desarrollo curricular. hacia las condiciones que le marcan el medio en el cual actian, ya que éstos logran sentido formativo cuando tienen relación directa con las circunstancias en las 


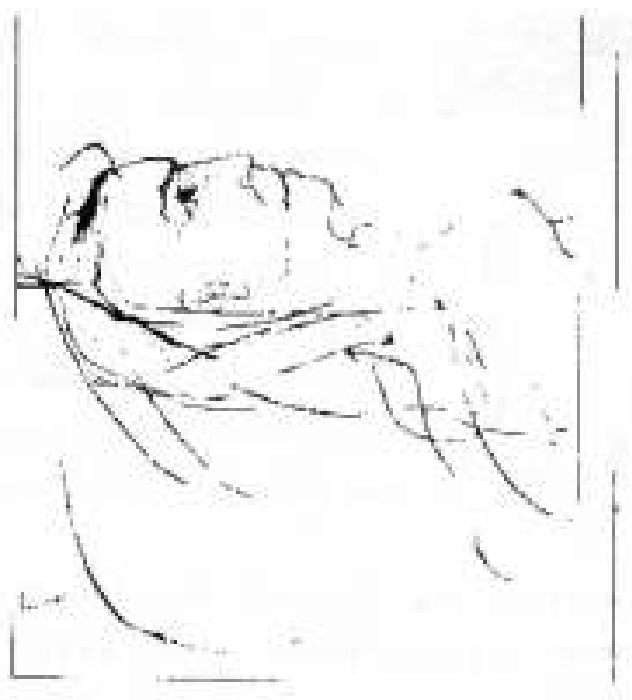

cuales se desenvuelve la realidad social y con los intereses de los educandos. Por ello, se sugiere que sus resultados y sus logros deben ser confrontados con las condiciones en las cuales se producen.

Lo dicho permite plantear que la labor desarrollada por las instituciones educativas, relacionadas con las diversas formas de saber y de cjecutar de las diferentes disciplinas, debe tascender las aulas y vincularse a la vida cotidiana de sus profesionales. de aquellas personas que están en proceso de capacitación y de los sectores de la población que son depositarios de su acción. De esta manera. los actores del proceso educativo pueden tener la oportunidad de crecery de construirse en lo personal y en lo social, a partir de las experiencias que obtengan de sus relaciones sociales. Un buen espacio para ello, son las actividades de proyección social generadas y desarrolladas por las actividades de origen académico.

De esto, fácilmente se desprende la necesidad y la posibilidad de que las personas que se desempeñan desde las instituciones educativas de nivel formal, tengan la oportunidad de elaborar y confrontar sus conceptos, sus fundamentos teóricos, desarrollar actitudes, valores, aptitudes y destrezas, apoyados en los acontecimientos que se presentan en la sociedad. Lascomunidades, aportaran sus vivencias, sus experiencias, sus saberes y además de ello. serán beneficiarias de lo que se realice en su medio, puesto que son perceptoras directis de las actividades. prácticas, conocimientos y habilidades desarrolladas por los académicos.

Tanto las personas que provienen de las instituciones educativas así como las personas de las comunidades pueden construirse mutuamente, teniendo como escenario lascondiciones reales en las cuales se ejerce la vida cotidiana de ambas. Esta perspectiva invita a entender que las prácticas acadèmicas deben trascender la función de "Extensión" planteada para las instituciones educativas, puesto que no se trata simplemente de vincularse a la sociedad. sino de ejercer en ella como parte integrante de su propia realidad y siendo participe de su propio desarrollo.

También se trata de reconocer que las personas que llevan a cabo trabajo comunitario a partir de una labor académica, si bien son parte de la sociedad y deben comportarse como tales, presentan su propia identidad y poseen caracteristicas particulares que los hacen diferentes de la población en donde actúan. Por tanto, no se debe pensar en que los educandos sean "iguales" a los miembros de la población. se trata más bien de reconocer las diferencias y de asumirlas como un factor importante para aprovechar los aportes mutuos que puedan hacerse. Esto debe tenerse en cuenta para efectos de entender el sentido de las prácticas académicas y la labor que con ellas se puede llevar a cabo, de tal manera que haya la posibilidad de orientar adecuadamente este tipo de actividades. No sobra decir que en esta perspectiva. el sector educativo se astume como un factor importante en los procesos de construcción de una sociedad que actue al servicio del hombre. 
Esto nos ha llevado a compartir los conceptos y opiniones de quienes plantean la necesidad de llevar a cabo acciones cducativas que sean altemativas frente a las vrientaciones de la pedagogia tradicional, dado que buscan apoyarse en el fomento de la acción social comunitaria y entiendan la educación como parte de los procesos de socialización de la especie humana. esta propuesta conceptual. Ileva implicita la necesidad de comprender que la escolaridad es sólo una parte de la formacion acadenica y que esta debe asumirse como parte del proceso social para que no se quede en la transmisión de contenidos posiblemente aislados del contexto social.

Se trata entonces. de que la labor educativa esté al servicio de la realización particular de los individuos y de la construcción de la sociedad en su conjunto, trascendiendo los programas escolares de corte tradicional. Ante todo, la formación académica, debe generar procesos de Desarrollo Humano, en los cuales predomine la intención de promover la construcción de actitudes. valores, saberes, reflexiones, capacidades, aptitudes, habilidades a nivel de las personas. y de promover cambios y transformaciones en el desarrollo social del pais, de las regiones y de las localidades, en los cuales la base sean acciones a nivel comunitario de corte participativo.

Las reflexiones que se originan a partir de este tipo de prácticas y la sistematizacion de experiencias ha permitido entender que las acciones educativas en las cuales se privilegien los procesos de proyección social. pueden convertirse en alternativa educativa en la medida en que se apoyen en los siguientes supuestos:

- Contextuar la labor educativa de acuerdo con las circunstancias y necesidades que determine la realidad social.

- Tener encuenta que la labor desarrollada a partir de las prảcticas académicas es llevada a cabo por personas y con ellas. por lo tanto. ésta cobra sentido cuando el interés fundamental de las acciones se centra en buscar el crecimiento de! nivel de Desarrollo Humano de los participantes.

- Orientar la función de los participantes en las prácticas académicas (Instituciones educativas. alumnos, profesores y población perceptora), hacia la construcción de la sociedad. mediante la generación y fortalecimiento de procesos de Desarrollo Social.

EL IMPACTO de las vivencias y de las experiencias que se han consiruido a partir de estas propuestas han incidido en las prácticas academicas y en los resultados de acciones de equipos de trabajo, tales como los conformados en la Facultad de Fisioterapia de la Universidad Autōnoma de Manizales. en el áreat de "Proyección Comunitaria". En esta Facultad participa un equipo interdisciplinario compuesto por doce (12) docentes y aproximadamente 150 alumnos. correspondientes a III. IV. V y VI semestres. Estas personas se desempeñan en 17 barrios de la ciudad de Manizales y se hallan vinculadas a los programas de cerca de 30 instituciones. También ejercen en una vereda del municipio de Neira (Caldas). en coordinación con el programa -Una Nueva Iniciativa Rural". UNIR, agenciado por la Universidad Autónoma de Manizales.

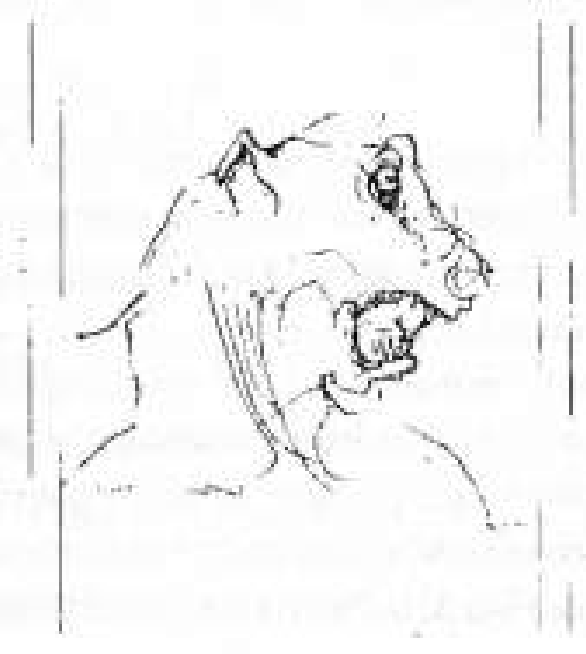




\section{«...el ejercicio de su función de ser parte del proceso de transformación social y de la búsqueda del desarrollo integral de estudiantes, profesores y de la sociedad de la cual hacen parte. Funciones que simultáneamente, y por ello mismo, constituyen el contenido de sus posibilidades de transmitir y de crear conocimientos».}

Los resultados de la labor de estos equipos de trabajo han permitido percibir interesantes alcances a nivel del crecimiento personal de los participantes, en cuanto a laconstruoción de saberes, actitudes, valores. habilidades destrezas. la generación de procesos de participación y de autogestión comunitaria, de autonomia. etc.

Se piensa que estos logros tienen que ver conel énfasis que se ha hecho en cuxinto a la necesidad de contextuar los contenidos de la acción educativa en las condiciones que presenta la realidad social y de apoyarse en los intereses y motivaciones de los alumnos practicantes. De la misma manera, las instituciones y grupos sociales han tenido la oportunidad de desarnollar algunos de sus proyectos sociales comunitarios y de lograr mayor eficacia y efectividad a nivel de la superación de las necesidades que presentan las poblaciones en las cuales actuan."

Tal como puede observarse. este texto remite a pensar el asunto en términos de lo educativo. quizás como parte del desarrollo curricular particular de una lacultad. pero es de reconocer que actualmente se trata de ver más alli. puesto que hay que mirar el papel del colectivo institucional en relación con el colectivo social. Es claro, entonces, que el marco de referencia debe trascender la mirada hacia el punto de vista de la Proyección Universitaria،antes que de la Proyeoción social vista desde las prácticas sociales estudiantiles que pueden ser uno de sus matices, pero que son limitadas frente a los alcances y posibilidades de incidir en lo macro social. Esto no quiere decir que la trascendencia no se dé en lo micro, sino que ésta no debe quedar desligada del conjunto institucional, dado que las misiones y las visiones deben ser orientadas por el colectivo institucional.

Precisamente. muxhas de las limitaciones que se han tenido para lograr un desarrollo efectivo de la propuesta originada en lo curricular. a nivel particular de un área de formación profesional, tienen que ver precisamente con la necesidad de respaldar las acciones particulares de las Facultades y/o de áreas con el compromiso institucional, aqui la universidad en su conjunto asume como posibilitadora de la realización de esta perspectiva.

Al mismo tiempo. muchos de los alcunces han tenido que ver con la concepción y compromiso que han orientado a los actores de estas experiencias. (profesores, estudiantes, instituciones, poblaciones. etc.) en la perspectiva de contribuir al desarrollo de la sociedad, aportando a ello. desde to micro. procesos de organización y participación comunitaria, interacción comunicativa, intercambio de saberes. gestión y administración de proyectos sociales concertados y negociados, y ante todo. 
vivencias y experiencias que han contribuido al desarrollo humano critico y ético de los participantes. Esto permite decir que la Proyección Universitaria debe ser una perspectiva de la Universidad en su conjunto. en la cual convergen los diferentes aspectos de la formación: investigación, formación profesional y proyección comunitaria. por lo tanto es más que un asunto de un área, llảmese Proyección Comunitaria uotra.

Lo anterior-permite pensar que la proyección social vista desde las prácticas estudiantiles se queda corta frente a las demandas de la sociedad, en cuanto a la necesidad de ser participe de los procesos de transformación de la realidad social en la cual se desempeña. puesto que requieren un respaldo y un compromiso institucional para lograr mayor incidencia en la vida social colectiva.

Para efectos de entender el papel de la universidad desde esta perspectiva. veamos lo planteado por Alejandro Acosta al considerar la Proyección Social de la Universidad como:

"El ejercicio de su función de ser parte del proceso de transformación social y de la búsqueda del desarrolls integral de estudiantes, profesores y de la sociedad de la cual hacen parte. Funciones que simultaneamente, y por ello mismo, constituyen el contenido de sus posibilidades de transmitir y de crear conocimienos $=$
Lo anterior y las experiencias que se han tenido al respecto en nuestro medio llevan a pensar que se hace necesario realizar cambios en los puntos de vista acerca de las relaciones de la universidad con la sociedad, el sector productivo y el Estado. para efectos de reorientar las acciones. teniendo como base el compromiso y la necesidad urgente de ser participe de las condiciones de vida de las poblaciones, de las relaciones sociales en las cuales se inscribe la función institucional. Se trata de climinar intencionalmente la distancia que se ha establecido entre la academia y la sociedad, que es lo que ha impedido que su labor sea de impacto en el medio. Esto nos permite acercarnos a lo indicado en la Facultad de Fisioterapia cuando expresan:

\footnotetext{
- La Universidad debe traseender su posicion tradicional sobre la Proyeccion como mera prestación de servicios y soluciones parciales a necesidades aisladas. en muchos casos descontextualizadas de la realidad social. hacia la busqueda de estrategias que identifiquen problemas y contribuyan a la solucion de hechos seciales $y$ a la vez faciliten lit vehiculizacion de la investigaciòn, que posibiliten la labor critica no solo de los educandos sino de los docentes dentro de la realidad concreta. Esto significa que las Proyección Sucial de la Universidad debe ser el proceso mediante ef cual se logre la transformación de la cultura, con visión futurista, proceso centrado en las necesidades de la region y cl pais" +
}

Es destacable el inter's por que se realicen acciones integradas, orientadas a detectar y transformar

\section{«... la Proyección Social de la Universidad debe ser el proceso mediante el cual se logre la transformación de la cultura, con visión futurista, proceso centrado en las necesidades de la región y del país».}


situaciones que afecten la región y el pais; sin embargo, se debe tener en cuenta que la mirada de la Proyección del Universidad, también debe trascender mucho de lo indicado en este párrafo, dado que la labor de la Universidad incluso debe ir más allá de lo académico y penetrar las diferentes esferas de la sociedad, eso si. desde lo académico que es su función desde la identidad que tiene Alejandro Acosta, al respecto indica:

"La universidad tiene que verse a si misma como parte del desarrollo social, como una estrategia de desarrollo social, en la medida en que, unida a las comunidades, al sector económico y al público produce conocimiento básico y aplicado relevante para las necesidades y el momento, lo transmite y lo aplica en un proceso integrado y continuow.

\section{IMPLICACIONES DE LOS CONCEPTOS DE EXTENSIÓN, PROYECCIÓN SOCIAL Y PROYECCIÓN UNIVERSITARIA PARA LA UNIVERSIDAD AUTÓNOMA DE MANIZALES}

\subsection{La Extensión Universitaria}

La extensión, como función de la universidad, deriva de varias circunstancias, entre ellas, es destacable la desarticulación de los procesos educativos con respecto a la realidad social en la cual están inscritos, lo cual ha conducido al deterioro creciente de los vinculos entre las instituciones académicas y la vida cotidiana de las personas. Las funciones educativas se asumen como procesos de escolaridad y por ende se privilegia la transmisión y recepción de información como formas de contribuir al desarrollo intelectual de los estudiantes. Esto ha conducido entre otros aspectos a:

Tal como se indicaba antes, se apoya en una visión fragmentada de la realidad, lo cual se manifiesta en la conservación de la dualidad entre teoria y práctica, asumiendo las instituciones educativas como centros del saber y lo externo como el espacio de la práctica. Para vincularse a esta última, se hace con base en la extensión o en el deseo de ampliar sus funciones.

Existe divorcio entre los diferentes sectores de la vida social del pais, por ejemplo la universidad y el sector privado; se trata de buscar acercamientos entre ellos, dada la separación de la institución con respecto al contex to en el cual se desempeña.

Algunas de las caracteristicas de este tipo de función son las siguientes:

1. Las labores académicas se centran en los planes de estudio, los cuales se asumen como la base del currículo, es por ello, que las acciones de extensión se entienden a manera de complemento de la docencia y de la investigación.

2. Se realizan labores de asistencia y de beneficencia al servicio de las poblaciones a las cuales se acercan. De esta manera se muestra "el compromiso y la sensibilidad social" de las instituciones para con los beneficiarios. En otros casos, se presentautilización y hasta manipulación de las poblaciones.

3. Predomina el interés particular de las instituciones y la sociedad se entiende como un elemento 
extraño: to mismo ocurre con las poblaciones. éstas entienden que el "extensionista" tambièn es un ser ajeno en la vida de ellas, es el agente externo. En términos generales. son seres extranos entre si.

4. Las acciones institucionales responden a necesidades particulares, coyunturales, al margen de las condiciones que le ofrece el contexto en el cual se desenvuelve, En este sentido se desprende que la investigación, también se ve aisladadel medio. de ahí que su impacto sea bajo y por ende, poco haya tenido que ver con el desarrollo de la sociedad en su conjunto.

\subsection{La Proyección Social}

Desde este punto de vista, se entiende que la labor educativa es un proceso que se da a lo largo de la vida y que ésta cumple un papel de gran importancia para el desarrollo de las personas a nivel particular y de la sociedad a nivel general. Se trata entonces de generar ambientes que permitan que los individuos accedan a los conocimientos y aprendizajes básicos con el propósito de satisfacer las necesidades individuales y colectivas.

Asume que ei desarrollo humano debe trascender lo cognitivo y lo relacionado exclusivamente con la capacitación en aspectos especificos de las profesiones, por ello, enfatiza en la necesidad de generar procesos participativos que faciliten la formaciónde personas que ejerzan intencionalmente en la sociedad. como parte de ella.

Para que se presente la Proyección Social, se hace necesario que se favorezca el desarrollo de las siguientes condiciones:

1. Contextuar las funciones institucionales en las circunstancias históricas y culturales en las cuales ejerce, $y$ teniendo en cuenta que el conocimiento que maneje tenga implicaciones en el medio, es decir, se hace necesario que la Universidad harga uso de su saber para beneficio de la población. en últimas, se trata de que tenga perspectiva ética acerca del conocimiento. Esta seria una buena forma de resolver el marginamiento social de la universidad. Veamos to que se ha dicho al respecto:

* la Universidad ha perdido buena parte de sus correspondencia con los procesos sociales. económicos, politicos y culturales que viven los paises. La población requiere apoyos y alternativas para ufrontar condiciones sociales cada vez más dificiles y en las cuales las estrategias de sobrevivencia a las cuales se hizo referencia, no son suficientes y requicren del apoyo de la universidad, pari comprender mejor el origen de los problemas como para poder estructurar soluciones menos gravosas para su calidad de vida y màs alineadas con una perspectiva a futuro para el pais"

2. Pensar la práctica educativa como un espacio de socialización en el cual los sujetos participantes en ésta, tienen la posibilidad y la necesidad de formarse en las competencias que requiere lat sociedad para mantenerse y reproducirse. En este sentido, los contenidos de los aprendizajes deben ser orientados hacia la utilidad y al uso social. En este sentido, estamos de acuerdo con Alejandro Acosta cuando indica:

"Solo la práctica social permitirá al individuo trascender de la información al conocimiento, es decir, a aquel proceso que le permite apropiarse de la información 
para transformar su quehacer y sa realidad"

3. Asumir que la realidad en la cual se inscriben las instituciones es compleja y abarca las diferentes formas de organización que se han establecido en la sociedad; además; que cllas son parte del conjunto secial y que sus funciones son componentes del engranaje en que se desenvuelve la vida cotidiana del pais. De la misma manera, los sujetos particulares tambičn se encuentran inmersos en la diversidad de factores que hacen preserxia en el medioen el cual viven.

Se trata de tener en cuenta la pluralidad de situaciones y de interrelaciones que acomparian el ejercicio vital de las instituciones y de los sujetos que las conforman. para efectos de orientar sus procesos de acción social. Desde este punto de vista, la Proyeccion Suxial, debe contemplar que:

- solo zsta interacción con la realidad y con las comunidades permitira que, al tiempo que se logra un astentico desarrollo invelectual de los iovenes y docentes investigadores, pueden avanzar en su formación como setes humanos integrales. Dado que esta integralidad no se logra sollo con la adquisición de conocinientos. sino tambien con las destrezas. habilidades y comportamientos requeridos para aplicarlos, asi como con la apeopiación de los valores y las actaludes que les permitiran realizarse como seres humanos de manera individual y por proy ección al contexto

4. Tener en cuenta que la construcción social de la sociedad es el resultado de la multiplicidad de interrelaciones entre las instituciones y los sujetos que las conforman. por lo tanto, no es un asanto de exclusividad de una de ellas en particular. De igual forma. los procesos de transformación social, tampoco son exelusivos de las instituciones. de ahí que en la búsqueda del desarrollo integral de estas y de los sujetos que participan de las comunidades que las conforman. deben ser entendidos como el resultado de acciones colectivas de cooperación e integracion. llaménse interprofesionales o interdisciplinarias. Esta mirada invita a que las instituciones actúen con sentido de lo colectivo. con un enfoque holistico. solidario, integral y cooperativo

5. Es importante que se tenga visión de futuro, es decir. que en su razón de ser se contemple la posibilidad de que puede suceder algo diferente de los acontecimientos del presente. sólo aquellos que creen en futuros deseables, tienen la posibilidad de concebir proyectos y de ser proyectivos. Se trata. no solamente de mantenerse, sino de construir perspectivas de tranformación tanto a nivel del colectivo social. como de la institución en particular. En este sentido, contemplan la posibilidad de adecuar su marcha de acuerdo con las circunsiancias en las que se desenvuelve su existencia. siempre en la mira de hacer realidad los sueños y esperanzas que se diseñan.

En torno de reflexiones similares, algumos colegas confirman lo expresado. cuando inviuan a tener en cuenta en los procesos de Proyección Social que:

\section{“...la Universidad ha perdido buena parte de sus correspondencia con los procesos sociales, económicos, políticos y culturales que viven los paises. "}


"Los procesos de construcción humana y de desarrollo de un pais se dan en contextos de interaciom entre actores, sectores, instituciones. etc. y se operan a través de la integracion de tsfikeraos, escenarios, y procesos de acción que ocienten el logrs de metas comunes, procesos en los cuales fo unversidad se provecta y se construye $=$

\subsection{Proyección Universitaria}

La Proyección Universitaria, no es simplemente un cambio de nombre, es otra mirada, otra forma de pensar y de actuar de las instituciones universitarias. con respecto a su función social, con el compromiso y la responsabilidad que tienen con respecto de la sociedad. Ello también implica que se adopte un punto de vista de desarrollo humano integral. en el cual lo social deja de ser un agregado para que se asuma como parte intrinseca del sujeto participante de la vida no sólo de la institución. sino de la sociedad.

El referente contextual tiene que ver con la manera como viene funcionando la sociedad en nuestro pais. por ello. se hace necesario remitirse a las orientaciones que se establecen en las tendencias que vivimos actualmente, no sólo en lo social en general. sino en lo educativo a nivel especifico. Esto no lleva a decir que la tendencia de la educación para el siglo XXI en nuestro pais se deriva y se ve reflejada en las diferentes normas que han emergido en la década de $10 s 90$ del siglo XX. entre ellas se puede expresar que la Constitución de 1991. las leves 30 de 1992 y 115 de 1994. especifican y coinciden en la necesidad de reformar la educación. en el sentido que las instituciones educativas tengan como finalidad:

-asumir la universalidad del conocimiento en la parficularidad de los contex tos socion-culturales que

\section{«... sólo esta interacción con}

la realidad y con las

comunidades permitirá que,

al tiempo que se logra un

auténtico desarrollo

intelectual de los jóvenes y

docentes investigadores,

pueden avanzar en su

formación como seres

humanos integrales.»

le dan existencia a la Nación come tal, en la diversidad de las regiones y en las autonomias de sus comunidades -y comprender la proyección social y cultural de la Escuela cono un "Proyecto de Vida"

Esto permite decir que las universidades no son instituciones cualesquicra que cumplen funciones al servicio de la sociedad pero en la especificidad de su quehacer, inclusive cada universidad tiene su propio sentido, su propia razón de ser. Veamos lo que indica Antanas Mockus en el primer caso:

\footnotetext{
"la misión de la universidad es deherse ante todo a si misma. Estoy diciendo algo que parcét extraño. la misión de la universidad seria ser fiel en todo sentido a si misma. obedecerse asi misma como tradición y como proyecto" "I
}

Lo indicado. nos permite inferir que la I/niversidad comoente particular se dehc a si misna. es autónoma y como parte del tejido social, se halla inmersa en la sociedad de la cual forma parte $y$ ante la cual debe responder por sus actos. es decir que su acción debe ser responsable.

En términos generales se puede deducir que la 
Proyección Universitaria es parte del andamiaje que han construido las instituciones universitarias a lo largo de su existencia, es un asunto de identidad y por ende. cada una de ellas proyecta su acción en tèrminos de lo que es y de aquello que se considera que permite construir su futuro, orientado por sus intenciones. finalidades y propósitos. No sobra indicar que a la base de esto están los criterios que le han dado respaldo y sentido a su existencia. su filosofia, sus concepciones antropologicas. pedagógicas y estatutarias.

Dado que la Provección Universitaria se basa en algunos conceptos. se trata de mirar la manera como estos pueden o no tener aplicabilidad en nuestro medio. dependiendo de las intencionalidades especificas de la institución. Veamos qué puede implicar este término, dado que se apoya entre otros aspectos en:

". el concepto de educación para todos,... el cual en terminos de la universidad implica el reconocimiento a que la educación no se vive soblo en sus claustros y salones. Requiere de la interacción y la convergencia con los espacios no escolares, lo cual. en csincidencia con la función de la universidad, exige que su proyección a los espacios no escolares se asuma como zinu funcioin

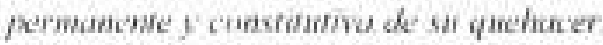

Implica también reconocer que la educación no se puede centrar en el desarrollo de curricula predisentados en las instituciones universitarias, a los cuales debe responder el joven universitario independientemente de sus caracteristicas de sujeto, de su entorno o del grado de pertinencia cultural que tenga.

El proceso tiene que centrarse en las necesidades reales del pais, en las de la región, en las caracteristicas del contexto cultural y en las condiciones especificas de los estudiantes y los profesores: como base para apropiarse de los saberes nacionales $e$ internacionales respectivos ${ }^{+4}$

En otras palabras y apoyándonos en el punto de vista de Borys Bustamente, se puede decir que la Proyección Universitaria, en términos generales. implica que en la práctica se propongan en las instituciones universitarias:

"nuevos niveles de organización, de selección. de administración de los contenidos escolares y de las relaciones de convivencias en la comunidad educativa articulado at un proyecto de una mejor sociedad"is

Pero no sólo de los contenidos académicos, quizís vistos desde la docencia. Se trata de que tengan y expresen puntos de vista claros con respecto al compromiso y a la responsabilidad yue tienen, dada su calidad de ser parte de la sociedad y no un simple agregado. por el contrario. es asumirse como parte del engranaje social. En este sentido. es de tener en cuenta lo indicado por Alejandro Acosta al respecto; veamos: 
Debe existir claridad en las universidades acerca de su función social y deben existir proyectos claris cuncretas cantinuas y xisfemánices que les permitan cumplir con dicha función" 14

Pero esa claridad y concreción de este tipo de proyectos a los cuales debe estar referida directamente la razon de ser de la universidad, debe estar planteada en términos de la función que le ha dado identidad no sólo en su origen, sino en su tradición y en las perspectivas de desempeño. se trata de noolvidar que es función de la universidad:

* . contribuir a recuperar ef saber y la cultura regional y kical, a apoyar su reafirmación y su enriquecimiento al convertirla en contenido del diálogo entre el saber de las diferentes disciplinas y el de las propias comunidades. Diálogo que tiene que darse en el proceso de adelantamiento de proyectos de investigación y de desarrollo en tomo a los cuales el curriculo deje de ser solamente transmision de informacion, de lectura y de repetición de lextos anticuados, para convertirse en verdadera estrattegia de desamollo social en la cual el conocimiento se construye integrando el saber local con el universal, en la solución de problemas concretos hasicos"it

A propósito de los proyectos que le dan sentido a la Proyección Universitaria. se hace necesario expresar que esta función de la universidad, hace referencia no solamente los que apuntan a lo externo en términos de mirar el contexto. por el contrario. debe ser preocupación suya las vivencias de la comunidad educativa puesto que la identidad se construye tambien en lo interno. Un ejemplo de ello debe ser el proyecto de Bienestar Universitario. el cual debe orientarse ante todo a contribuir a la construcción de pertenencia y velar por la calidad de vida óptima de los diversos sectores que hacen parte de su comunidad.

Lo indicado antes, invita a que se haga referencia directa al pensamiento que ha manifestado la U.A.M. al respecto, dado que en ello se puede percibir la tradición y la identidad. requisitos hásicos para establecer la perspectiva de Proyección y p $\mathrm{w}$ ende. la manera de orientar la Provección Universitaria especifica de la U.A.M.

En algunos de los objetivos académicos de LA UNIVERSIDAD AUTONOMA DE MANIZALES sepuxten percibir los puntos de visti expresados en torno a ta orientación que se le debe dar ala Proyección Universitaria. Entre otros. estos son:

*a. Preparar ciudadanos integrales para que mediante la formación cientifica y profesional que reciban en la UNIVERSIDAD AUTONOMA DE MANIZALES, puedan cumplir sur propósitos personales, familiares y sociaies.

c. Ser factor de desirrsllo cientificu, cultural. económico, politico y ético a nivel nacional y regional.

5. Promover la unidad nacional, la descentralización. la integración regional y la cooperación institucional con miras a que las diversas zonas del pais dispongan de los recursos humanos y de las tecnologias apropiadas que les permitan atender adecuadamente sus necesidades.n

En este mismo sentido, en el Estatuto Profesmal. se plantea en el articulo 4 :

"Se denominan actividades de serfessirin a" 
praseccian aquellas que estan constituidas por programas especiticos. dirigidos a los miembros de la Universidad o a personas $e$ instituciones externats, de carácter curricular o extracurricular. siempre bajo responsabilidad de la Universidad:"

Aunque teminológicamente aparecen como si fuera lo mismo extensión y proyección, aún en el sentido se puede percibir el predominio del concepto de extensión. dado que además de expresarse como actividades, éstas se enmarcan dentro de programas especificos. donde se capta la orientacion hacia lo particular de su campo de acción. Es bueno recondar que aurque las acciones de Proyección Universitaria se manifiestan en programas o proyectos, más que ¿́stos, está en su base una visión de la función social que debe tener la universidad. Por si hay dudas. esto se reafima en lo indicado en el articulo 4. del fstatuto Gieneral. en el cual se indica:

\section{LIA UNIVERSIDAD AUTONOMA DE MANIZALES tendrà como objeto fundamental la educación superior a sus mas altos niveles: el desarrollo de las ciencias, la filosofia y las artes y la extensión educativan.}

Según lo expresado, se hace importante que se piense que en este momento la posición de la universidad ha venido variando en términos de adecuarse a las demandas de la sociedad contemporanea. de las responsabilidades que derivan de nuevas formas de concebir la función universitaria. Esto puede percibirse en la Misión de la U.A.M. cuando en ella se plantea que:

"Somos una comunidad educadora, dinamizadora
del conocimiento, comprometida con la convivencia
pacifica y el desarrollo, que contibuye a ta
formación de personas éticas, con pensamiento
crítico e innovador"

De lo cual se derivan. los criterios para expresar cada uno de los términos empleados y las implicaciones conceptuales y prácticas de cada uno de ellos.

"Comunidad Educadora: Somos un grupo social conformado por profesores. estudiantes. egresados, funcionarios y demais colaboradores de la Universidad. cuyos intereses e identidad consisten en buscar formas nuevas de generar desarrollo mediante el aprovechamiento del conocimiento cientifico y tecnológico en la creación de ambientes de convivencia. Desde esta perspectiva, la U.A.M. es una institución de educacion superior.

Dinamizadora del C'onocimiento: Somos capaces de apropiar. crear y transformar el conocimiento que posibilite asumir el cambio con responsabilidad ética, politica y cientifica para propiciar el bien común.

Compromerida con la Comsivencia Pacifica: Somos defensores de la vida y de los valores.

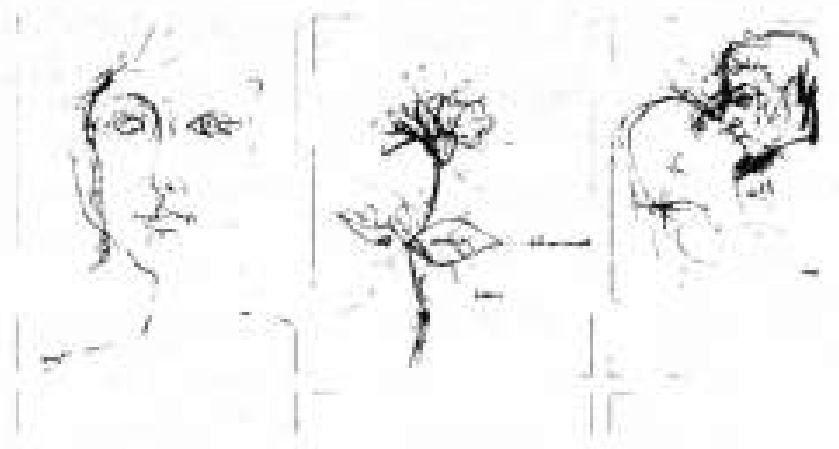


potenciamos creativamente los recursos existentes, promovemos la conciencia social. formamos en la libertad responsable $y$, apoyados en la capacidad de pensar en forma reflexiva, de argumentar y contraargumentar, fomentamos el entendimiento en la pluralidad, respetando la diversidad.

Desarrollo: La U.A.M. como institución social busca el mejoramiento de la calidad de vida de las personas y de los grupos para lograr un desarrollo regional sustentable.

Contribuye a la formación de personas éticas, con pensamiento crítico e innovador: $\mathrm{La}$ U.A.M. ayuda a la persona a formarse para aprender permanentemente y para que en el cjercicio de su autonomia, y como sujeto social. sea capaz de tomar posición frente al conflicto y reorientar las acciones particulares y colectivas haciael bien individual y social. Coneste propósito construimos procesos innovadores que nos permiten crear nuevos horizontes de actuación local y global, mirando las experiencias. conocimientos previos y sus posibilidades de realización efectiva para construir un futuro mejor. ${ }^{\text {"th }}$

Dada la claridad acerca de la razón de ser actual. de la universidad, debe ser la referencia necesariae imprescindible para efectos de expresar las orienta- ciones conceptuales y prácticas relacionadas con la PROYECCIÓN UNIVERSITARIA ENLA UNIVERSIDAD AUTÓNOMA DE MANIZALES. por ello se propone que esta se entienda como:
"UNA FORMA DE CONCEBIR. ORIENTAR Y REALIZAR ACCIONES POR PARTE DE LA COMUNIDAD QUE CONFORMA LA U.A.M. INSPIRADA EN UNA PERSPECTIVA DE DESARROLLOHUMANOQUEHACEÉNFASIS ENLA FORMACIÓN DE PERSONAS ETICAS, CRITICAS E INNOVADORAS. EL. CONOCIMIENTO SE APROVECHA Y SE. UTILIZA CONLA INTENCIÓN DE CONSTRUIR AMBIENTES DE CONVIVENCIA PACIFICA TANTO A NIVEL, INTERNOCOMOEXTERNO DELAINSTITUCIÓN, CONLOQUESE BUSCA PROPICIAR EL BIEN COMÚN Y EL MEJORAMIENTO DE LA CALIDAD DE VIDA DE LAS PERSONAS, EN EL MARCO DE UN DESARROLLOREGIONALSUSTENTABLEQUE CONTEMPLE PROCESOS SOCIALES. EOONOMMICOS. CULTURALES Y POLITICOS.

Este concepto implica varias posturas de indole epistemológica, teórica y metodológica que propenden al desarrollo humano integral, holistico. histórico, dinámico de los procesos que se realizan en el contexto de la vida de la institución.

Implicar:

Establecer relación directa con la investigación. dado que requiere identificar los problemas

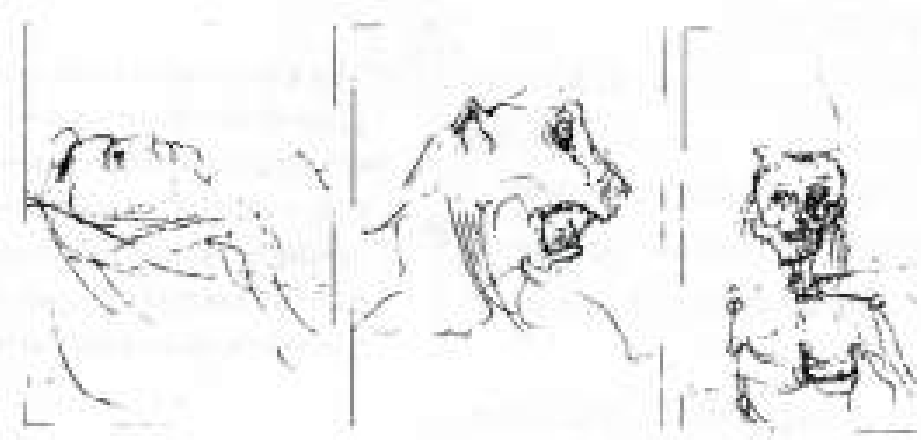


reales, para efectos de elaborar propuestas que atiendan efectivamente las problemáticas que afecten el medio en el cual estamos inscritos.

Partir del criterio que los conocimientos son aplicables a la realidad y que ésta es una instancia importante para efectos de establecer la validez de los mismos.

Comprometerse con la idea y la intención de contribuir a la construcción de la cultura del pensamiento critico, innovador y creativo.

Asumir. como parte de su responsabilidad social, la necesidad de contribuir a la formación de personas éticas, es decir, de generar y consolidar valores mediante los cuales se privilegie la proyección de las prácticas institucionales al medio social, en aras de contribuir a la convivencia pacifica y al desarrollo de la sociedad. .

Propende a la interdisciplinaridad y al trabajo en equipo, dado que se considera que ni el saber, ni ta realidad en la cual actúa son exclusivos de las personas y las instituciones.

Orientar esfuerzos hacia la construcción de macroproyectos que logren impactar la realidad social en la cual estamos inmersos.

Exigir que las universidades tengan una orientación definida frente a lo que quieren respecto a la proyección y a la investigación, en términos de su participación en la vida política. social. cultural y económica de la sociedad colombiana.

Enmarcar su acción en las políticas educativas, a partir de su misión institucional.

Entender que su acción es colectiva, hacerla desde su identidad. lo cual se logra con base en el reconocimiento de la diferencia con respecto a los demás. La identidad está en que se origina en una institución académica, no se diluye en lo social

Entrar a ser parte de la vida cotidiana de otras poblaciones y con clias construir comunidad. Asumir que el conocimiento es el eje de sus acciones sociales.

Entender que los conocimientos y saberes que se manejan en la universidad tienen que ver con los que se manejan en otros sectores de población.

Considerar que la universidad cumple un papel importante en el desarrollo social del pais y que del desarrollo del pais depende su futuro, por ello, se compromete con la vida de la sociedad. Asumir que no se puede actuar al margen del desarrollo del pais, ni a espaldas de él.

Propender al manejo de conocimientos que respondan a las condiciones de la realidad en la cual nos desenvolvemos

Percibir la necesidad de adecuar sus contenidos a las demandas de la sociedad.

Entender la pertinencia de la universidad en el desarrollo cultural de la sociedad, tanto en lo local, lo regional, nacional e incluso intemacional. La Proyecciòn Universitaria vista como un proyecto politico, se materializa en acciones de la universidad, respaldadas en el compromiso ético - social en que fundamentan su razón de ser.

La Proyección Universitaria vista como un proyecto pedagógico, lleva a construir procesos de formación en los cuales su comunidad educativa aprende del entomo, en el entomo y con el entorno.

"La universidad tiene que verse a si misma como parte del desarrollo sociat, como una estrategia de desarrollo social, en la medida en que, unida a las comunidades, al sector económico y al público produce conocimiento basico y aplicado relevante para las necesidades y el momento, lo transmite $y$ lo aplica en un proceso integrado y continuo ${ }^{\text {in }}$ 
1) Contiene aportes importantes de ia Doctora. Francia Restrepo de Mejia, de la Doctora. Luz Guiomar Maldonado Perez y los profesores Jorge Eliécer Rodriguez Giraldo, José Armando Vidarte Claros, Juan Pablo Garcia y Jeremias Quiñones Cärdenas.

2) CASTILLO Garcia. José Rubén, "Manizales: Ciudad Educadora" En Revista ANFORA. Universidad Autónoma de Manizales, año 4, nùmero 8 , juliodiciembre de 1996, pảg. 84 y 85.

3) CASTIL LO Garcia, José Rubèn. "Por qué la Proyección Comunitaria en la Educación Actual?. En: FISIOCIENCIA, Revista de la Facultad de Fisioterapia de la Universidad Autónoma de Manizales. Año I No. 1. 1994 . Pag 12-15,

4) ACOSTA, Ayerbe Alejandro. De la Extensión Universitaria a la Proyección Social de la Universidad. Ponencia, Santafé de Bogota, Julio de 1993.

5) COMITE DE CURRICULO DE LA FACULTADDE FISIOTERAPIA. Proyección Social de la Universidad. En: ANFORA. Revista de la Universidad Autónoma de Manizales, afho 6, Número 12, Octubre-Diciembre de 1998 y Enero de 1999 , Pàg. 50

6) ACOSTA Ayerbe Alejandro. Op. Cit. Pig. 12

7) ACOSTA, Ayerbe Alejandro, Op. Cit. Pág 14.

8) ACOSTA Ayerbe Alejandro. Op. Cit. Päg, 14

9) ACOSTA, Ayerbe Alejandro. Op. Cit. Pág. 17.

10) UNIVERSIDAD DE MANIZALES. "Bases Conceptuales y Contextuales de la Proyección Social en la Universidad de Manizales". Mimeografiado. S. N.T.

11) MOCKUS, Antaras, "Fundamentos Teóricos para una Reforma de la Universidad" en la Universidad Hoy. Revista Educación y Cultura, Pàg. 23.

12) ACOSTA, Ayerbe Alenjandro. Op. Cí. Paig, 19

13) BUSTAMANTE, Borys. "Pensar el Curriculo", S, N T.

14) ACOSTA, Ayerbe Alejandro. Op, Cit, Päg 20.

15) ACOSTA, Ayerbe Alejandro. Op. Cit. Pag 21

16) UNIVERSIDAD AUTONOMA DE MANIZALES, folleto de presentación institucional.

17) ACOSTA, Ayetbe Alejandro. Op. Cit. Pike 12.

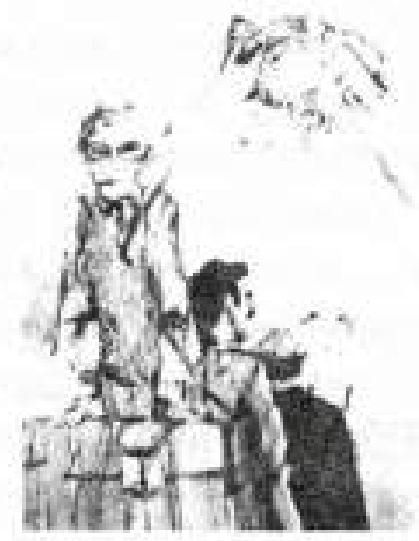

